\section{Fungal development}

The Filamentous Fungi. Vol. 3: Developmental Mycology. Edited by J. E Smith and D. R. Berry. Pp. 464. (Edward Arnold: London, 1978.) $£ 24.50$.

Over the years, the attractive and often bizarre range of morphological forms produced by filamentous fungi have proved irresistible to individuals ranging from illustrators of the earlier mycological texts to, more recently, overly self-indulgent scanning electron microscopists. The attractions have not gone unnoticed by developmental biologists, a band whose ranks have recently been swelled by molecular biologists ruefully contemplating the day when Escherichia coli, with a final intake of its terminal electron acceptor. yields its last molecular secret. And this is where a basic problem lies. The molecular biologist is superbly equipped to study the molecular basis of development in biological systems, such as $E$. coli, which are easy to manipulate in the laboratory. But filamentous fungi are anything but easy to work with in the laboratory. Attractively shaped differentiated structures they certainly produce, but isolating fractions rich in these structures, a necessary preliminary to a study of their molecular architecture, is often virtually impossible. Nevertheless, the molecular basis of differentiation in filamentous fungi has for many years had its coterie of practitioners. Developmental Mycology, the third volume in a series entitled The Filamentous Fungi, is a progress report up to and including 1976 on the activities of this cavalier band.

The editors took a very broad view of the term 'fungal development', taking license to include hyphal growth. This proved to be a bonus for, in molecular terms, this is undoubtedly the best understood aspect of the subject. At the same time, they have adhered strictly to filamentous fungi, with the result that a discussion of differentiation in the cellular and acellular slime moulds is omitted.

In all, there are 22 chapters, the first third of the book containing chapters on various aspects of hyphal growth. The chapters on fungal protoplasts and on mathematical analysis of growth are highly recommended, and both make a valuable contribution to the literature. But the dust jacket on the book tells us that the text aims to provide an integrated perspective of fungal development. Why then did the editors commission separate chapters on the cytology and enzymology of hyphal tip growth? Both are good accounts, but one chapter bringing together both aspects of the subject would surely have more long-term value.

The middle section of the book deals with fungal developmental processes that lead to formation of structures that are morphologically different from hyphae. This section starts with a very readable chapter on yeast-mycelium dimorphism. Admittedly, the authors were able to call on a large body of experimental data but, by concentrating on fundamental concepts. they have produced a most valuable article. The balance between cytology and biochemistry that one would ideally like to see in the remaining chapters is, however, all too obviously missing. In general, this is no fault of the authors. but (as already outlined) results simply because the systems being studied are not amenable to sophisticated biochemical examination.

The book ends with chapters on fungal development induced by light and temperature, circadian rhythms, cell ageing and autolysis, cytoplasmic inheritance and senescence and fungal development and metabolite formation. reflecting yet again the broad view of developmental mycology taken by the editors. Although some of these subjects, such as production of secondary metabolites, have recently been thoroughly reviewed elsewhere, others including circadian rhythms and cell ageing have not, and so are exceptionally welcome.

\section{Cerebral potentials}

Progress in Clinical Neurophysiology. Vol. 3: Language and Hemispheric Specialization in Man: Cerebral EventRelated Potentials. Edited by J. E. Desmedt. Pp.286, (S. Karger: Basel, 1978.) SFr./DM98; \$43.75.

A PROMINENT problem in neurobiology is to specify the properties of the speech systems of the human brain. Why are they alone capable of elaborating speech and language? We do know that it is not merely on account of their inflow and outflow; the comparable region of the 'subordinate' or non-speaking cerebral hemisphere has the same connexions. yet that region is involved in sustaining language only up to $10-14 \mathrm{yr}$ of age and cannot thereafter become involved. We do not know why language generally resides in only one cerebral hemisphere; why this should be the left hemisphere; nor yet why language develops readily in human children but only partially and after laborious training (if at all) in apes.
The publishers make the sweeping claim that the contributions to the book "go beyond the standard review-type article by developing concepts in cach area of Developmental Mycology", Some. particularly those that describe aspects of hyphal growth, do, whereas others do not and indeed could not because of the paucity of information available on many differentiation processes. When one turns, however, to other biological developmental processes, particularly those in prokaryotic microbes, one has seen over the years the emergence of fascinating, if at times contentious, new concepts such as commitment, long-lived $m R N A s$ and regulation through modification to enzyme structure. Developmental Mycology would have been richer as a text if these concepts had at least been introduced, and their possible role in fungal development speculated on. However. the only attempt at an introduction to basic concepts is a tiny six-page chapter which does scant justice to the concepts of differentiation.

But these are not major shortcomings. Developmental Mycology is a welcome and timely volume which should be available to all who work with filamentous fungi, and not just those whose interests are in fungal development.

A. H. Rose

A. H. Rose is Professor of Microbiology at the University of Bath, UK.

The majority of the more than 30 contributors to this book are only peripherally concerned with the neural bases of language. They have described a variety of temporal relationships between the mass electrical potentials of the brain and linguistic or other performance. The potentials themselves, rather than the behavioural processes. seem to have become their main preoccupation. Indeed, new findings on the potentials are reported. Unfortunately the mass potentials afford an imprecise index of the eiectrical activity of the brain. (crtainly they serve to lateralise language functions to one hemisphere, thus confirming the earlier clinical and experimental observations. And the more informative methods of recording from single brain cells cannot be applied to man. So we have to accept even a small step forward. one that is unlikely to reveal any truly fundamental new knowledge, with the reassurance that in the hands of these particular expert contributors the limits of the usefulness of this approach have now probably been reached. G. Ettlinger

$G$. Ettlinger is Professor of Neuropsychology at the Institute of Psvchiatry. University of London, UK. 